\title{
Molarenextraktion bei Heimtieren - Indikation und Technik
}

\author{
Stefan Gabriel
}

Veränderungen an den Backenzähnen und deren Folgeerkrankungen wie Granulome und Abszesse haben in der Heimtierpraxis eine große Bedeutung. Zugleich sind sie der Diagnostik und Therapie schwer zugänglich. Die elodonten Backenzähne bei Kaninchen und den Nagern (Meerschwein, Chinchilla, Degu) stellen wegen ihrer besonderen Anatomie eine große Herausforderung an die Extraktionstechnik dar.

\section{Zahnerkrankungen}

Kleinnager (Maus, Ratte, Hamster) haben normal bewurzelte, schmelzhöckerige kleine Backenzähne (Prämolaren und Molaren), die selten auffällig werden. Aufgrund ihrer omnivoren bzw. granivoren Ernährungsweise neigen sie zu Karies und Zahnabnutzung, Frakturen und Zahnverlusten. Solche Zähnchen gehen meist unbemerkt verloren und werden wohl äußerst selten zur Extraktion vorgestellt.

Die wurzeloffenen elodonten Zähne ( $\triangleright$ Exkurs) bei Kaninchen und den herbivoren Nagern Meerschwein, Chinchilla und Degu wachsen permanent mit hoher Eruptionsrate von 1-3 mm/Woche. Sie werden natürlicherweise durch intensives Mahlen strukturierten Grünfutters entsprechend abgenutzt. Bei unzureichender Kautätigkeit kommt es durch fehlenden Abrieb zu Zahnüberwachstum (Elongation). Diese entsteht durch fehlerhafte Fütterung mit schnell sättigendem Kraftfutter oder mangelhaftes Kauen infolge schmerzhafter Zustände. Pathologische Kaukräfte bei chronischer Malokklusion im Backenzahngebiss führen zu Veränderungen an den Zähnen selbst und Lageveränderungen aus der Zahnreihe heraus. Typische Veränderungen sind Zahnspitzenbildung, Deformationen und Verbiegungen. Bei chronischer Malokklusion kommt es durch permanenten pathologischen Druck auf die Zahnwurzeln zu entzündlichen Prozessen mit retrograder Wurzelverlagerung, Granulombildung und zu Zahnwurzelabszessen.

\section{Diagnostik}

Die intraoralen Aspekte der Backenzähne lassen sich durch Inspektion mit dem
Trichterotoskop oder dem Video-Endoskop nur unzureichend einsehen. Bei vollständiger Exploration der Maulhöhle in Narkose nach Einsetzen von Kieferund Wangenspreizern können pathologische Zahnspitzen, Zahnverbiegungen und -dislokationen diagnostiziert werden. Da aber der größte Teil des Backenzahns im Kiefer liegt, können der gesamte Zahn und seine Umgebung erst durch die Röntgendiagnostik beurteilt werden.

Die Diagnostik am wachen Tier hat bei Backenzahnproblemen lediglich indizierenden Charakter. Erst durch die Untersuchung der gesamten Maulhöhle in Narkose sowie eine gute Röntgendiagnostik können die Backenzähne sicher beurteilt werden.

Eine überlagerungsfreie Darstellung aller Backenzähne ist am Schädel des Kleinsäugers nicht möglich. Daher müssen hochauflösende Röntgenbilder ( $\mathbf{A b b}$. 1, - Abb.2) bei standardisierter Lagerung in mehreren Ebenen angefertigt werden, um Zähne und Kiefer sicher beurteilen zu können. Die in diesem Artikel dargestell-

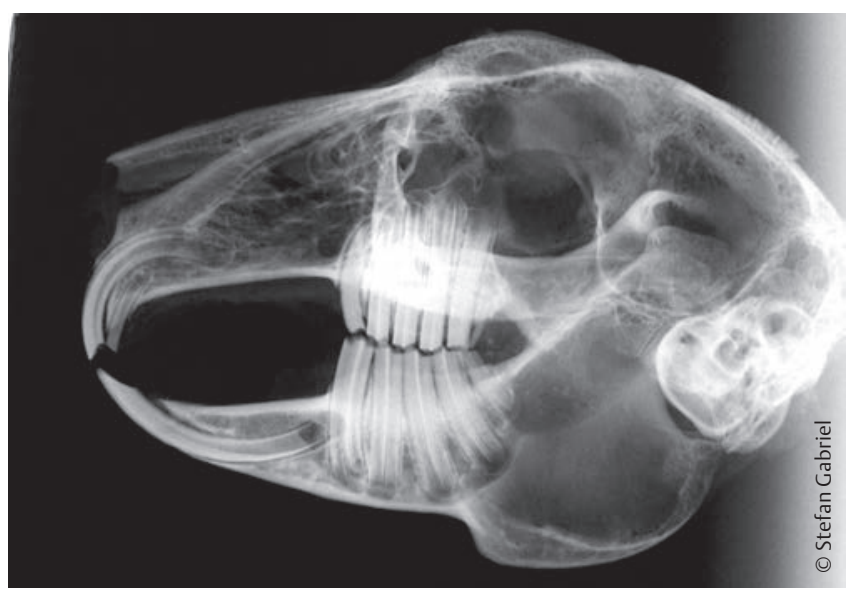

Abb. 1 Hochauflösendes Röntgenbild eines Kaninchens in laterolateraler Projektion („Panorama“-Ansicht). Halbschädelpräparat.

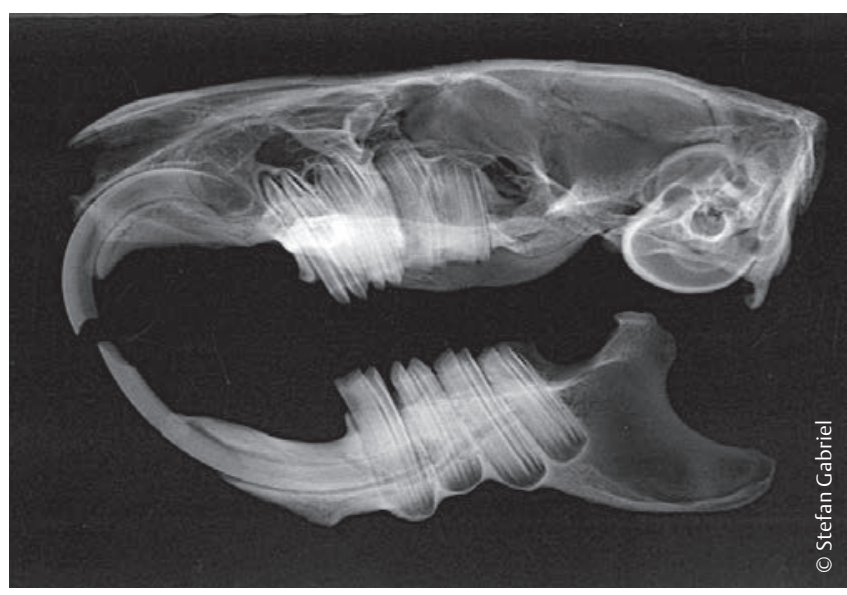

Abb. 2 Hochauflösendes Röntgenbild eines Meerschweinchenschädels in laterolateraler Projektion. Halbschädelpräparat mit ausgelöster Mandibel zur überlagerungsfreien Darstellung. 


\section{Exkurs}

\section{Anatomie und Pathologie elodonter Zähne}

Anders als festverwurzelte Zähne stecken elodonte Zähne mit einer faserigen Wurzelscheide in der knöchernen Alveole. An der apikalen Wurzelöffnung wird durch die germinativen Zahngewebe Schmelz und Dentin gebildet. Der Zahn wächst permanent nach und eruptiert koronal aus der Alveole, um sich in der Okklusionsfläche an den Antagonisten abzureiben. Prämolaren und Molaren sind morphologisch gleich und bilden die funktionelle Einheit der Backenzahnreihe. Winkelung und Relief der Kauflächen sind tierartspezifisch.

Aufgrund ihrer anatomischen Lage ragen die ersten Prämolaren $\left(\mathbf{P}_{2}\right)$ im Oberkiefer beim Kaninchen in die Nasenhöhle. Raumfordernde Prozesse an diesen Zahnwurzeln können den benachbarten Tränen-Nasen-Kanal komprimieren und zur Stase des Tränenabflusses sowie zur Dakryocystitis führen.

Die letzten Prämolaren $\left(\mathbf{P}_{4}\right)$ und die vorderen Molaren $\left(\mathbf{M}_{1}\right.$ und $\left.\mathbf{M}_{2}\right)$ der Oberkiefer liegen mit ihrer Wurzel in der Orbita und können bei Wurzelentzündungen zu retrobulbären Abszessen führen.

Im Unterkiefer führen retrograde Wurzelverlagerungen zu Auftreibungen, Perforationen und Abszessen. Diese Entzündungsreaktionen können enorme Ausmaße annehmen ( $\mathbf{A b b}$.4), weil die Ursache des Abszesses persistiert. Häufig wachsen die Molaren sogar in ausgeprägten Zahnwurzelabszessen ganz oder teilweise weiter ( $\bullet$ Abb. 7), was ohne Röntgenkontrolle nicht zu beurteilen ist.

ten CT-Bilder wurden experimentell mit einem Mikro-CT aus der Werkstoffprüfung an Knochenpräparaten angefertigt ( $\vee$ Abb.3). Sie sind in ihrer Auflösung nicht mit den üblicherweise vorhandenen klinischen CT-Bildern vergleichbar. Gerade für die feinen und feinsten Veränderungen an Zähnen und Kiefern ist die klassische (Dental-)Röntgentechnik immer noch unübertroffen [5]. Zur isolierten Darstellung der Unterkieferäste wird eine Schrägprojektion mit einer Kippung von etwa $40^{\circ}$ verwendet.

\section{Indikationen}

Eine Indikation zur Extraktion besteht bei folgenden Veränderungen:

- vereiterte Molaren, die als Auslöser von Kieferabszessen identifiziert wurden

- mobile, parodontal gelockerte Backenzähne
- Tränennasenkanal-Stenose beim Kaninchen

- retrobulbäre Abszesse

Mobile, parodontal gelockerte Backenzähne führen zu einer Gewebszerstörung, die sich durch eine Alveolitis mit Auflösung des Parodonts, alveolären Knochenschwund und Osteomyelitis äußert. Eine Restitutio ad integrum ist daher auch unter Antibiotikaeinsatz unmöglich. Solche Zähne stellen meist unbemerkt die Ursache größerer Abszessbildungen dar. Bei Kaninchen mit Tränennasenkanal-Stenose wird man einen pathologischen Prämolaren $\mathrm{P}_{2}$ zur Druckentlastung extrahieren. Bei retrobulbären Abszessen sind meist retrograd entzündete Molaren $\left(\mathrm{M}_{1}\right.$ oder $\left.\mathrm{M}_{2}\right)$ ursächlich und zu entfernen.

Häufig wird angefragt, ob man einen „störenden“ Backenzahn, z.B. wegen sublingualer Spitzenbildung, nicht einfach ziehen kann. Dazu ist zu bemerken, dass sich ein wurzelgesunder Molar nicht aus der Zahnreihe entfernen lässt, ohne die Nachbarzähne zu schädigen. Das gelingt höchstens bei den endständigen Molaren. - Abb. 5 zeigt die bilophodonte Innenstruktur der Molaren. Dies bedeutet, dass ein zusammengesetzter elodonter Zahn aus 2 nebeneinander angeordneten und verbundenen Zahnkörpern besteht, von denen jeder ein eigenes Pulpenorgan besitzt.

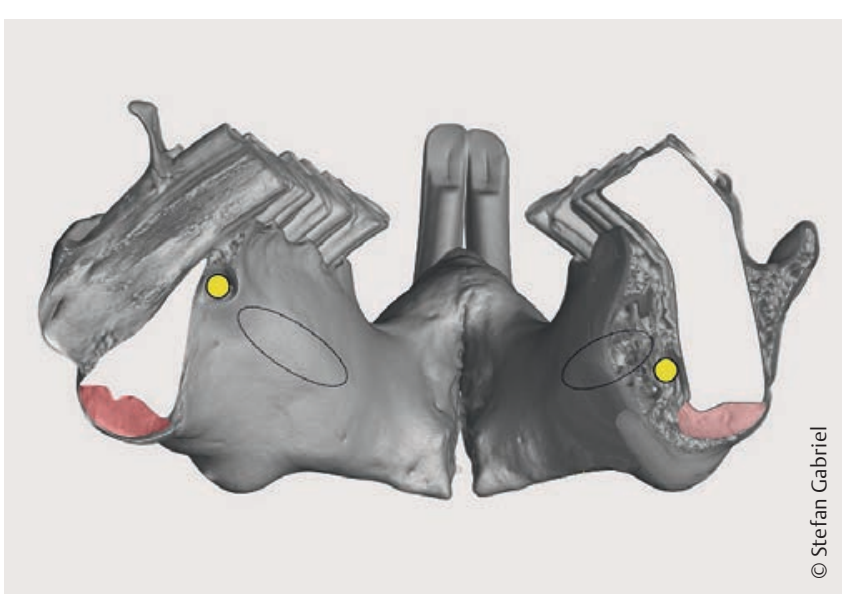

Abb. 3 3D-Rekonstruktion eines Mikro-CT des Unterkiefers eines Meerschweinchens mit Schnitt in Höhe des 2. Molaren (weiße Fläche). Ansicht von kaudal. Beachte die Lage der Inzisivenwurzeln (Ovale), des Canalis mandibularis (gelb) und des germinativen Zahngewebes bzw. der Zahnwurzel (rot).

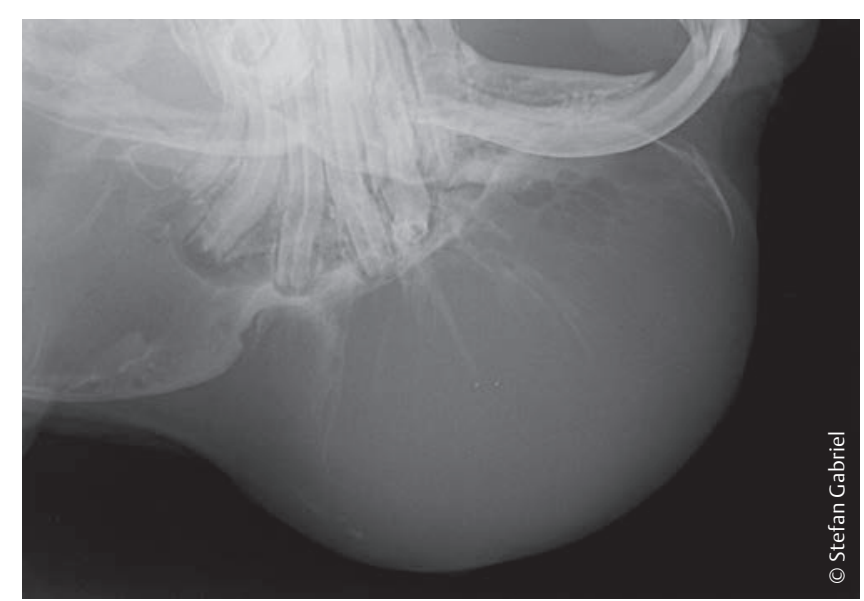

Abb. 4 Röntgen-Schrägaufnahme eines Kaninchens mit Unterkieferabszessbildung. Zur überlagerungsfreien Darstellung der plattennahen linken Mandibel wurde der Schädel mit $40^{\circ}$ von unten angestrahlt. Beachte die Wurzelveränderung des ersten Prämolaren $\left(\mathrm{P}_{3}\right)$ als Ursache des Abszesses und die knöcherne Reaktion des Periosts (Spiculabildung). 


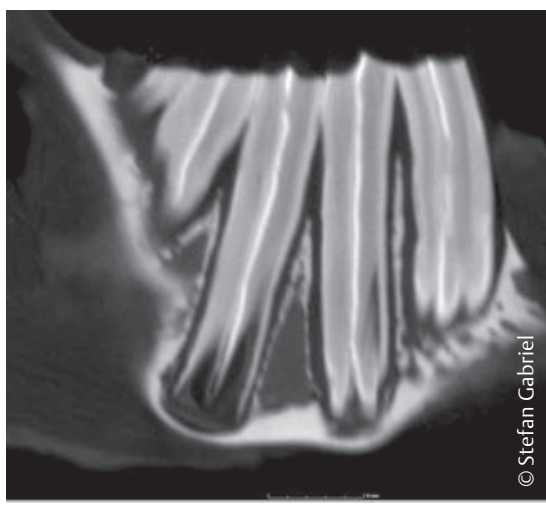

Abb. 5 Mikro-CT: Mandibel eines Kaninchens im Längsschnitt. Beachte die bilophodonte Innenstruktur der Backenzähne und die geschlossene Zahnreihe auf der Kaufläche mit dem arttypischen Relief.

Bei veränderten Molaren ( Abb.6, - Abb. 7) besteht immer die Gefahr, dass der Zahn beim Extraktionsversuch aufgrund von Schwachstellen in seiner Substanz (Locus minoris resistentiae) frakturiert. Bei einzelnen veränderten Molaren innerhalb der Molarenreihe besteht die Problematik, dass die Stabilität der Zahnreihe verloren geht und die verbleibende Zahnlücke Folgeprobleme bereiten wird. $>$ Abb. 7 demonstriert sehr eindrücklich die Problematik, dass nach Abszessbehandlungen mit Entfernung des oder der ursächlichen Zähne oft riesige Wundhöhlen zurückbleiben. Alveolen im Oberkiefer haben nach Zahnextraktion eine bessere Heilungstendenz als im Unterkiefer, weil die Schwerkraft die Wundreinigung begünstigt. Zahnlücken im Unterkiefer heilen grundsätzlich schlechter ab, weil Futterpartikel eingepresst werden und der Detritus nicht von selbst abfließen kann.

\section{.konkret}

Zahnextraktionen im Oberkiefer haben meistens eine bessere Heilungstendenz als im Unterkiefer.

\section{Technik}

Vor der Entfernung eines Backenzahns gilt es stets sehr sorgfältig abzuwägen und zu bedenken, dass nach jeder Extraktion eine oral schlecht heilende Alveole zurückbleibt.

\section{Orale Molarenextraktion}

Das Prinzip ist die allseitige Lockerung durch Durchtrennung der parodontalen Fasern mithilfe kleiner Luxatoren. Dies ist wegen der beengten Verhältnisse in der Maulhöhle, der eingeschränkten Sicht und den fragilen Strukturen oft technisch schwierig. Der Fachhandel bietet einige Modelle an, da man aber verschiedene Größen und Winkelungen benötigt, wird man um eine kreative Selbstherstellung nicht herumkommen. Böhmer empfiehlt bei kleinen Zähnchen die Verwendung abgeknickter Injektionskanülen [1, 2].
In den $>$ Abb. 8 und $\triangleright$ Abb. 9 wird der Einsatz des Luxators am Oberkiefer $\mathrm{M}_{2}$ demonstriert. Der passende Luxator wird an allen Seitenflächen in den Parodontalspalt eingeführt und mit wiegenden Bewegungen in die Tiefe der Alveole gedrückt. Das Instrument wirkt dabei als Keil, der den Zahn nach lateral hebelt. Die scharfe Schneide zertrennt parodontale Fasern. Nach möglichst tiefer Lösung des Parodonts wird der Zahn durch interdentales Hebeln und vorsichtiges Rotieren um seine Längsachse weiter gelockert, bis er in Richtung seiner Längsachse herausgezogen werden kann ( Abb. 10). Im Unterkiefer ist diese Manipulation wegen der steileren Zahnstellung deutlich schwieriger. In der Enge der Maulhöhle muss der Molar manchmal während der Extraktion vorsichtig geteilt werden, damit man mit der Fasszange nachfassen und tiefer ansetzen kann.

Cave
Niemals sollte man einen unzureichend
gelockerten Backenzahn mit Gewalt
extrahieren.

\section{Extraorale Molarenextraktion}

Der chirurgische Zugang erfolgt manchmal bei deformierten sowie bei Extraktionsversuchen frakturierten Zähnen. Dazu wird nach einem Hautschnitt und einer Präparation der Weichteile der

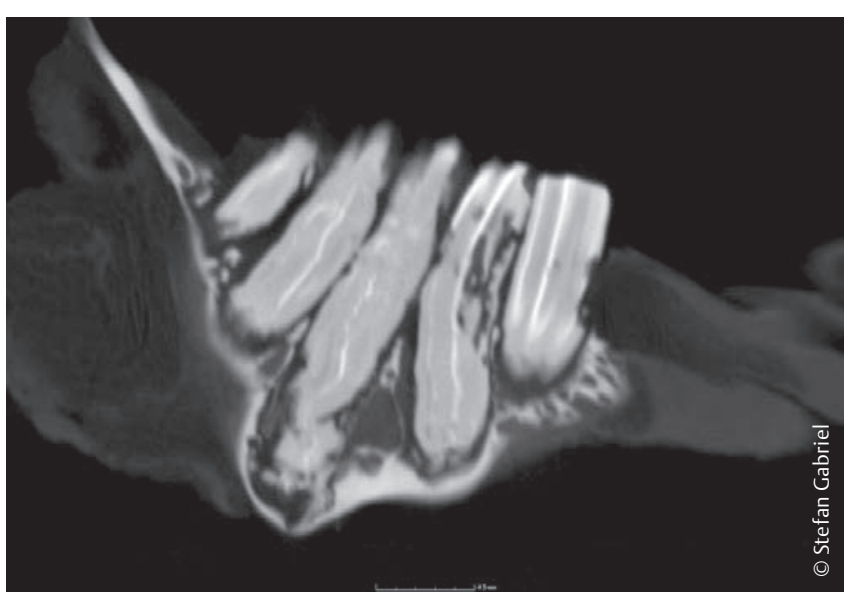

Abb. 6 Mikro-CT: Mandibel eines pathologischen Unterkiefers (Kaninchen). Beachte die hochgradig veränderten Innenstrukturen, die Wurzelveränderungen, den Schwund des Alveolarknochens und die coronalen Zwischenräume.

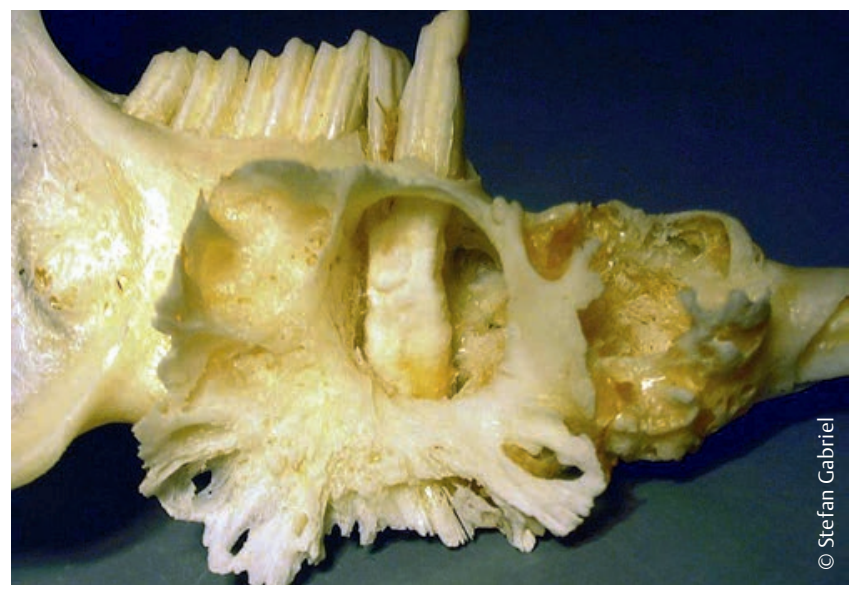

Abb. 7 Unterkieferpräparat eines Kaninchens mit chronischen Unterkieferabszessen. Beachte den ausgefallenen abszedierten Inzisivus (rechts) und die Spaltung des P3. Da der Zahn offensichtlich trotz Abszessbildung wochenlang weitergewachsen ist, ist die Spaltung mit der Fasereinspießung sicher sekundär. Auslöser war wahrscheinlich ein Übergreifen des Wurzelabszesses vom Inzisivus. 


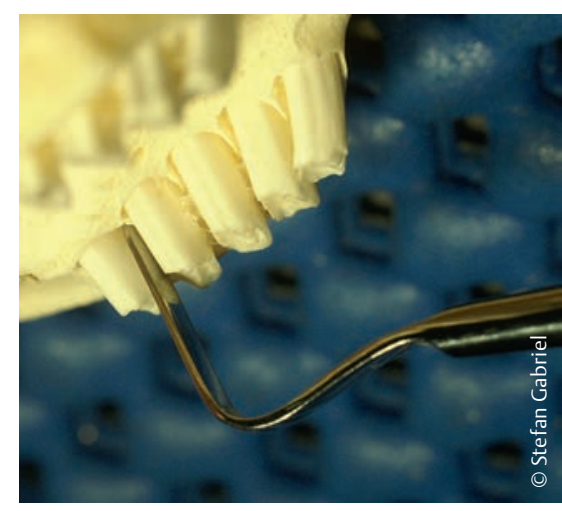

Abb. 8 Demonstration der Molarenextraktion am Knochenpräparat. Der Luxator (angeschliffener Heidemann-Spatel) wird interdental eingebracht.

Wurzelbereich unter Schonung der A. und V. facialis dargestellt und die Alveole mit einer Knochenfräse eröffnet ( $\vee$ Abb. 11). Der Zahn oder der Wurzelrest wird dann retrograd herausgedrückt. Dieses invasive Verfahren bietet sich an, wenn gleichzeitig ein Abszess entfernt werden muss.

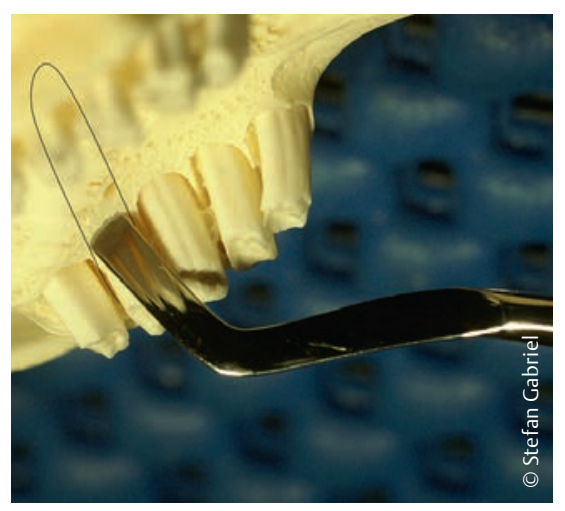

Abb. 9 Die laterale Lösung erfolgt durch Einführen des Luxators in gleicher Weise. Beachte die angeschliffene Schneide an der Spitze des Luxators. Der Verlauf des Molaren $\mathrm{M}_{2}$ ist eingezeichnet.

\section{Nachbehandlung}

Nach erfolgter Extraktion wird der Zahn zunächst auf Vollständigkeit untersucht. Beim Vorliegen eines Wurzelgranuloms tritt oft spontan Eiter aus. Auftretende Blutungen sind in der Regel durch kurze

\section{Praxistipp}

Luxationswerkzeuge können aus Heidemann-Spateln verschiedener GröBe und Winkelung selbst hergestellt werden, indem man die Spatelspitze mit der Diamantscheibe scharf anschleift. Als Fasszangen eignen sich parallel greifende Arterienklemmen mit unterschiedlicher Biegung oder die Fasszange nach Crossley.

Kompression mit einem Stieltupfer zu beherrschen.

Bei retrobulbären Abszessen kann nach der Molarenextraktion von der Alveole aus die Abszesshöhle mittels Curettage und Spülung (PVP-Jodlösung) drainiert und gereinigt werden. Zur Vermeidung einer Aspiration von Eiter oder Spüllösung ist der Zungengrund sorgfältig zu tamponieren. Es ist darauf $\mathrm{zu}$ achten, dass der Abfluss aus dem Maul bei nach unten gebeugtem Kopf gewährleistet ist. 


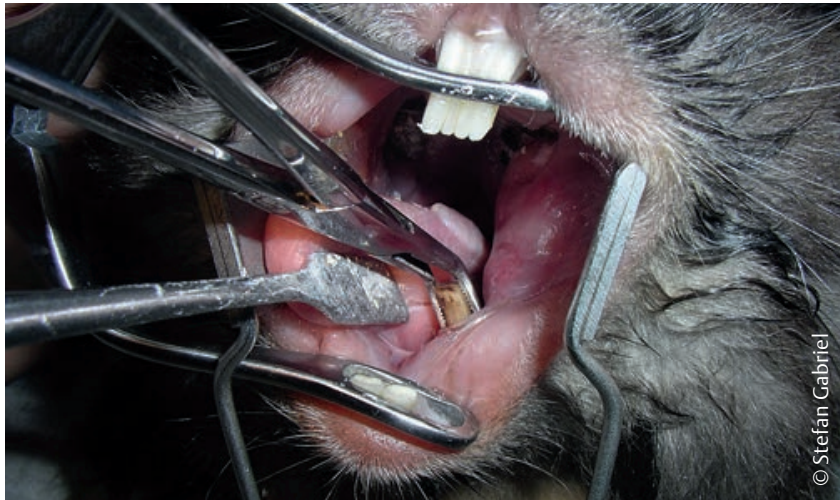

Abb. 10 Der losgelöste erste Prämolare im Unterkiefer $\left(\mathrm{P}_{3}\right)$ wird mit einer passenden Zange (modifizierte Arterienklemme) so tief wie möglich erfasst und axial herausgezogen.

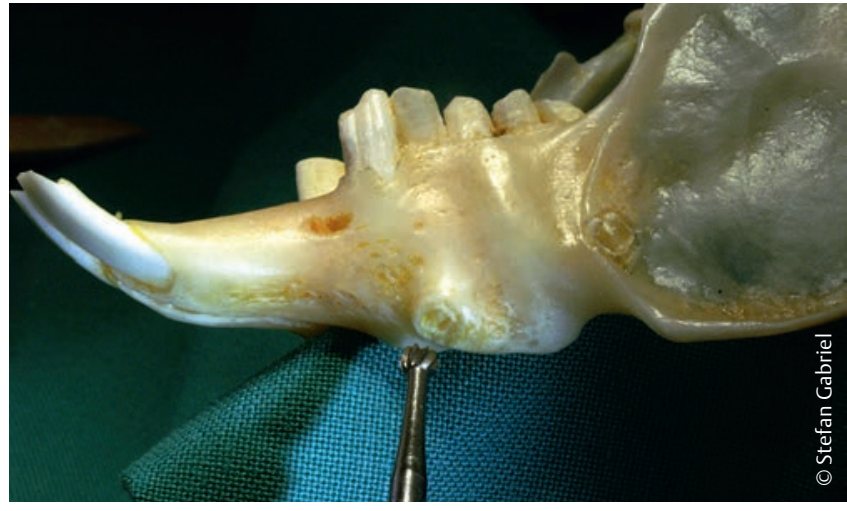

Abb. 11 Demonstration des chirurgischen Zugangs zu den Molarenwurzeln im Unterkiefer. Die Kompakta der Mandibel wird mit einem Kugelfräser entfernt und der Zahn $\left(\mathrm{P}_{4}\right)$ retrograd entfernt.
Manuka-Honig oder Chlorhexidingel haben sich als temporäre Einlagen bewährt.

\section{Cave}

Antibiotische Einlagen verbessern die Wundheilung nicht, können aber die Darmflora fatal schädigen.

\section{Oberkiefer}

Die Alveole bleibt offen, damit Sekrete abfließen können und die Wunde ausgranuliert. Nicht infizierte Alveolen heilen dabei in der Regel vollständig aus. Insbesondere der letzte Molar $\left(\mathrm{M}_{3}\right)$ und erste $\operatorname{Prämolar}\left(\mathrm{P}_{2}\right)$ haben eine gute Prognose.

\section{Unterkiefer}

Im Unterkiefer besteht die Problematik, dass Detritus und Futterpartikel nicht abfließen können und die Wundheilung verhindern. Unterkieferabszesse können so nach Zahnextraktion durch Futterpartikel und Bakterien aus der Maulhöhle aufrechterhalten werden, wenn es nicht gelingt, die Alveole zum Ausgranulieren zu bringen. Dies ist besonders bei Extraktion oder Verlust mehrerer Molaren problematisch. Eine Tamponade mit resorbierbaren Materialien kann versucht werden, eine Patentlösung gibt es bislang jedoch noch nicht. So ergibt sich relativ häufig die frustrierende Situation, dass nach Backenzahnextraktionen im Unterkiefer zwar der Auslöser entfernt werden konnte, eine aufwändige Nachbehandlung allerdings folgen muss.
Eine Abszessbehandlung kann nur erfolgreich sein, wenn die auslösende Noxe, also meist ein Backenzahn, restlos entfernt wurde. Vor einer halbherzigen Behandlung durch äußerliche Punktion und Spülung von Kieferabszessen ist ausdrücklich zu warnen. Diese rezidivieren zwangsläufig, solange die eigentliche Ursache nicht entfernt worden ist.

Postoperativ ist der Heimtierpatient bei guter Analgesie schnellstmöglich anzufüttern und sollte erst nach sicherer selbstständiger Futteraufnahme nach Hause entlassen werden. Bei Abszessen ist eine systemische Antibiose, möglichst nach Resistenztestung, nötig. Grundsätzlich sind eine Antiphlogese mit NSAID über mehrere Tage und vor allem eine Röntgenkontrolle nach etwa 4 Wochen anzuraten.

Der Patient muss nach einer Zahnextraktion regelmäßig kontrolliert und nachkorrigiert werden, da eine normale Zahnabnutzung nicht möglich ist. Die Extraktion des Antagonisten ist aber nicht sinnvoll, da jeder Zahn bei der Kaubewegung mit mehreren Gegenspielern okkludiert.

\section{Fazit}

Während die Inzisivenextraktion bei Kaninchen und Nagern ein probates und technisch relativ einfaches Mittel zur Beseitigung anders nicht zu korrigierender Inzisiven-Malokklusionen darstellt, muss man bei Backenzahnproblemen stets sehr sorgfältig abwägen und bedenken, dass nach jeder Extraktion eine oral schlecht heilende Alveole zurückbleibt.
Andererseits kann eine Abszessbehandlung nur erfolgreich sein, wenn die auslösende Noxe, also meist ein Backenzahn, restlos entfernt wurde.

Den Besitzern muss verständlich gemacht werden, dass diese Patienten immer wieder tierärztlich nachkontrolliert und gegebenenfalls auch nachbehandelt werden müssen.

\section{Online zu finden unter}

http://dx.doi.org/10.1055/s-0042-109907

\section{Literatur}

1 Böhmer E. Extraktion von Schneidezähnen bei Kaninchen und Nagern - Indikationen und Technik. Tierarztl Praxis 2003; 31 (K): 309-320

2 Böhmer E, Fahrenkrug P. Zahnheilkunde bei Kaninchen und Nagern: Lehrbuch und Atlas. Hannover: Schattauer; 2011

3 Crossley DA. Extraction of rabbit incisor teeth. J Brit Vet Dent Assoc 1994; 2: 8

4 Gabriel S. Röntgendiagnostik bei Malokklusion des Kaninchens. Veterinärspiegel 2013; 1: $17-21$

5 Gabriel S. Praxisbuch Zahnmedizin beim Heimtier. Stuttgart: Enke; 2016

6 Hartmann M. Zahnextraktion hypsodonter Zähne bei Nagern und Hasenartigen. Veterinärspiegel 2007; 1: 22-27

\section{Dr. Stefan Gabriel}

Tierärztliche Praxis für Kleintiere, Heimtiere, Zahnheilkunde

Le-Puy-Str. 13

59872 Meschede

dr.gabriel@t-online.de 